\title{
GENERAL LINEAR METHODS FOR VOLTERRA INTEGRO-DIFFERENTIAL EQUATIONS WITH MEMORY*
}

\author{
CHENGJIAN ZHANG ${ }^{\dagger}$ AND STEFAN VANDEWALLE ${ }^{\ddagger}$
}

\begin{abstract}
A new class of numerical methods for Volterra integro-differential equations with memory is developed. The methods are based on the combination of general linear methods with compound quadrature rules. Sufficient conditions that guarantee global and asymptotic stability of the solution of the differential equation and its numerical approximation are established. Numerical examples illustrate the convergence and effectiveness of the numerical methods.
\end{abstract}

Key words. stability, general linear methods, Volterra delay-integro-differential equation

AMS subject classifications. 65R20, 45L05, 65L20

DOI. $10.1137 / 040607058$

1. Introduction. Volterra integro-differential equations (VIDEs) arise widely in the mathematical modeling of physical and biological phenomena. Significant advances in the theoretical analysis of such equations and in the numerical analysis and implementation of time-integration techniques for these problems have been made in the last few decades. For a survey of early results we refer the reader to the books $[9,26]$. More recently, one has found that VIDEs with memory (MVIDEs), also called Volterra delay-integro-differential equations (VDIDEs), can be even more effective than standard VIDEs for the modeling of real-life problems; see, e.g., [8, 22]. This fact has led researchers to develop a theory and numerical analysis for MVIDEs. For example, numerical time-integration techniques of one-step collocation and RungeKutta (RK) type were investigated in $[10,17,11,23,34,21]$. Linear multistep based methods were studied in $[1,2,3,27,33]$.

So far, very few papers have discussed the nonlinear stability of MVIDEs. This is quite different from the case of delay differential equations (DDEs) without distributed delay, for which many results have been reported. For example, linear stability results for DDEs from [28] were extended in [29] to the nonlinear DDE case. In that paper it was proved that certain RK methods satisfy a so-called $B_{\rho}$-stability property. For the implicit Euler rule, [30] shows a contractivity result, which is a quite strong nonlinear stability property. Based on the concept of $B N_{f}$-stability, the authors of $[6,7,31,32]$ obtained a series of stability and contractivity results for continuous RK methods. In [32] it was shown that the requirement of $B N_{f}$-stability for a numerical method is a very strong requirement that leads to an order barrier. In order to avoid that order barrier, in $[19,20]$ numerical stability results have been derived for nonlinear DDEs based on the concept of algebraic stability of the underlying methods.

Stability results for MVIDEs were obtained in [5, 33, 34], where the authors investigated the nonlinear stability of continuous RK methods, discrete RK methods,

*Received by the editors April 20, 2004; accepted for publication (in revised form) May 19, 2005; published electronically February 3, 2006.

http://www.siam.org/journals/sisc/27-6/60705.html

${ }^{\dagger}$ Department of Mathematics, Huazhong University of Science and Technology, Wuhan 430074, Hubei, China (Cjzhang@mail.hust.edu.cn). This author's research was supported by Fellowship F/02/019 of the Research Council of the K. U. Leuven and by NSFC (No. 10571066).

${ }^{\ddagger}$ Department of Computer Science, Katholieke Universiteit Leuven, Celestijnenlaan 200A, B-3001 Leuven, Belgium (Stefan.Vandewalle@cs.kuleuven.be). 
and backward differentiation (BDF) methods, respectively. For a study of MVIDEs of neutral type we refer the reader to [12], where the concept of $B N_{f}$-stability is used as the basic assumption. For adaptations of RK methods towards general Volterra functional differential equations, we would like to point out the recent paper [25]. There, stability results have been derived based on $B$-stability of the underlying schemes. These results are applicable for time-integration on a finite time interval. Here, we will study the nonlinear stability of numerical schemes for MVIDEs based on the use of general linear (GL) methods. Our stability results will be applicable on infinite time intervals.

This paper is organized as follows. In section 2, a fairly general class of MVIDEs is defined. This class contains the problems considered in [33,34]. We present a stability criterion for such problems, which generalizes the criteria in the above references. In section 3, a class of extended GL methods is derived for solving MVIDEs. They are obtained by combining classical GL methods with compound quadrature rules. In section 4, some technical lemmas are derived. These lemmas will play a key role in the derivation of the numerical stability results. In sections 5 and 6 , we study the global and asymptotic stability of the extended GL methods. In section 7 we present some numerical examples in order to illustrate the convergence of the methods. These results show that the new methods are quite effective. Finally, in section 8 , we end with some concluding remarks.

\section{A class of MVIDEs and its stability.}

2.1. A general stability result. We consider a complex $N$-dimensional system of MVIDEs with constant delay $\tau>0$ of the form

$$
\left\{\begin{aligned}
y^{\prime}(t) & =f\left(t, y(t), y(t-\tau), \int_{t-\tau}^{t} g(t, v, y(v)) d v\right), & & t \in\left[t_{0},+\infty\right), \\
y(t) & =\varphi(t), & & t \in\left[t_{0}-\tau, t_{0}\right],
\end{aligned}\right.
$$

where the functions $f, g$, and $\varphi$ are smooth enough such that system (2.1) has a unique solution $y(t)$. We assume in particular that the following conditions are satisfied:

$$
\begin{aligned}
\Re\langle f(t, x, y, z)-f(t, \tilde{x}, \tilde{y}, \tilde{z}), x-\tilde{x}\rangle & \leq \alpha\|x-\tilde{x}\|^{2}+\beta\|y-\tilde{y}\|^{2}+\sigma\|z-\tilde{z}\|^{2}, \\
\|g(t, v, x)-g(t, v, \tilde{x})\| & \leq \gamma\|x-\tilde{x}\|, \quad(t, v) \in \mathbb{D},
\end{aligned}
$$

for $t \in\left[t_{0},+\infty\right), \mathbb{D}=\left\{(t, v): t \in\left[t_{0},+\infty\right), v \in[t-\tau, t]\right\}$, and $x, y, z, \tilde{x}, \tilde{y}, \tilde{z} \in \mathbb{C}^{N}$. The notations $\langle\cdot, \cdot\rangle$ and $\|\cdot\|$ denote a given inner product in $\mathbb{C}^{N}$ and its induced norm. The constants $(-\alpha), \beta, \sigma$, and $\gamma$ are nonnegative. Problems of type (2.1) with (2.2) and (2.3) will be called problems of class $\mathbb{G} \mathbb{R} \mathbb{I}(\alpha, \beta, \sigma, \gamma)$. Some examples will be given in section 7 . For problems of class $\mathbb{G R I}(\alpha, \beta, \sigma, \gamma)$, we can derive the following stability result.

TheOREM 2.1. Assume that system (2.1) belongs to the class $\mathbb{G} \mathbb{R I}(\alpha, \beta, \sigma, \gamma)$ with $\beta+\sigma \gamma^{2} \tau^{2}<-\alpha$. Then the following global and asymptotic stability properties hold:

$$
\begin{gathered}
\|y(t)-\tilde{y}(t)\| \leq \max _{\theta \in\left[t_{0}-\tau, t_{0}\right]}\|\varphi(\theta)-\psi(\theta)\| \quad \forall t \geq t_{0}, \\
\lim _{t \rightarrow+\infty}\|y(t)-\tilde{y}(t)\|=0
\end{gathered}
$$

where $\tilde{y}(t)$ is the solution of (2.1) with $\varphi(t)$ replaced by $\psi(t)$.

Proof. Denote by $\Delta y(t)$ the difference $y(t)-\tilde{y}(t)$. Using (2.2) and (2.3), we find 


$$
\begin{aligned}
& \frac{d}{d t}\left(\|\Delta y(t)\|^{2}\right)=2 \Re\left\langle\Delta y(t),(\Delta y(t))^{\prime}\right\rangle \\
& \quad \leq \alpha\|\Delta y(t)\|^{2}+\beta\|\Delta y(t-\tau)\|^{2}+\sigma\left\|\int_{t-\tau}^{t}[g(t, v, y(v))-g(t, v, \tilde{y}(v))] d t\right\|^{2} \\
& \quad \leq \alpha\|\Delta y(t)\|^{2}+\beta\|\Delta y(t-\tau)\|^{2}+\sigma \gamma^{2} \tau^{2} \max _{t-\tau \leq v \leq t}\|\Delta y(v)\|^{2} \\
& \quad \leq \alpha\|\Delta y(t)\|^{2}+\left(\beta+\sigma \gamma^{2} \tau^{2}\right) \max _{t-\tau \leq v \leq t}\|\Delta y(v)\|^{2} .
\end{aligned}
$$

Application of the generalized Halanay inequality from [4] leads to

$$
\|\Delta y(t)\|^{2} \leq \max _{\theta \in\left[t_{0}-\tau, t_{0}\right]}\|\varphi(\theta)-\psi(\theta)\|^{2} \forall t \geq t_{0} \quad \text { and } \quad \lim _{t \rightarrow+\infty}\|\Delta y(t)\|^{2}=0 .
$$

Thus, (2.4) and (2.5) are satisfied.

REMARK 2.2. The $\mathbb{G} \mathbb{R I}$ class includes the $\mathbb{D I}$ and $\mathbb{R I}$ problem classes that were investigated in $[33,34]$. The relation among these classes is as follows:

$$
\mathbb{D I}\left(\alpha, \beta,\left(\sigma_{1}, \sigma_{2}\right), \gamma\right) \subset \mathbb{R} \mathbb{I}\left(\alpha, \beta \sigma_{1}, \beta \sigma_{2}, \gamma\right) \subset \mathbb{G} \mathbb{R} \mathbb{I}\left(\alpha+\beta\left(\sigma_{1}+\sigma_{2}\right) / 2, \beta \sigma_{1} / 2, \beta \sigma_{2} / 2, \gamma\right) .
$$

The research in the framework of class $\mathbb{G} \mathbb{R I}$ extends the results from $[33,34]$.

REMARK 2.3. When the distributed delay argument is absent from (2.1), the problem becomes a DDE. In that case, Theorem 2.1 can be applied with $\gamma=\sigma=0$, which leads to the stability condition $\beta<-\alpha$.

2.2. Relation to earlier work. The result from Remark 2.3 can be related to earlier results on analytical stability of DDEs. In [28, 29], Reverdy dealt respectively with linear and nonlinear DDEs of class $\mathbb{G} \mathbb{R I}(-\rho, \rho, 0,0)$, with $\rho>0$. This class contains, for example, the linear DDE

$$
\left\{\begin{aligned}
y^{\prime}(t) & =p y(t)+q y(t-\tau), & & t \in\left[t_{0},+\infty\right), \\
y(t) & =\varphi(t), & & t \in\left[t_{0}-\tau, t_{0}\right],
\end{aligned}\right.
$$

with $\Re(p) \leq-|q|$, by setting $\rho=-\Re(p) / 2$. It was proved for $\mathbb{G} \mathbb{R} \mathbb{I}(-\rho, \rho, 0,0)$ that

$$
\mathcal{N}[y(t)-\tilde{y}(t)] \leq \mathcal{N}\left[y\left(t_{0}\right)-\tilde{y}\left(t_{0}\right)\right] \quad \forall t \geq t_{0},
$$

where $\mathcal{N}[y(t)]=\frac{1}{2}\|y(t)\|^{2}+\rho \int_{t-\tau}^{t}\|y(\theta)\|^{2} d \theta$. Note that stability property (2.7) follows from our somewhat stronger result (2.4).

In [30] Torelli considered DDEs with a variable delay $\tau(t)$ :

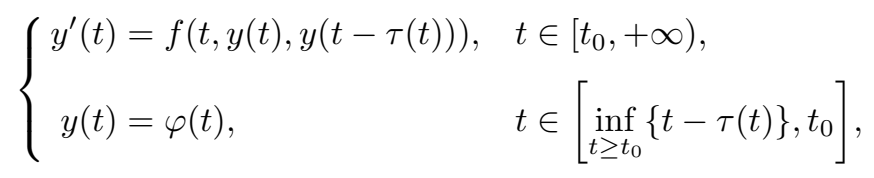

with $\beta(t) \leq-\alpha(t)$ and $\alpha(t)<0$ for $t \geq t_{0}$, where $\alpha(t)$ and $\beta(t)$ are defined as

$$
\begin{aligned}
& \alpha(t)=\sup _{x, \tilde{x}, y, \in \mathbb{C}^{n}, x \neq \tilde{x}} \Re\langle f(t, x, y)-f(t, \tilde{x}, y), x-\tilde{x}\rangle /\|x-\tilde{x}\|^{2}, \\
& \beta(t)=\sup _{x, y, \tilde{y} \in \mathbb{C}^{n}, y \neq \tilde{y}}\|f(t, x, y)-f(t, x, \tilde{y})\| /\|y-\tilde{y}\| .
\end{aligned}
$$

In that paper it was proven that (2.8) satisfies the global stability property (2.4).

System (2.8) was analyzed by Zennaro in [32] under a different set of conditions: 
(I) there exists a constant $\tau_{0}$ such that $\tau(t) \geq \tau_{0}$ for all $t \geq t_{0}$;

(II) the delayed argument $t-\tau(t)$ is a strictly increasing function for all $t \geq t_{0}$;

(III) $\lim _{t \rightarrow+\infty} t-\tau(t)=+\infty$;

(IV) there are two continuous functions, $\hat{\alpha}(t)$ and $\hat{\beta}(t)$, and two real numbers, $0 \leq r<1$ and $\alpha_{0}<0$, such that $\alpha(t) \leq \hat{\alpha}(t) \leq \alpha_{0}$ and $\beta(t) \leq \hat{\beta}(t) \leq-r \hat{\alpha}(t)$ for $t \geq t_{0}$.

It was proven in [32] that, under those conditions, system (2.8) satisfies the asymptotic stability $(2.5)$.

The above results by Torelli and by Zennaro can be applied to the constant delay case, i.e., with $\tau(t) \equiv \tau$. Assume there exists an $\alpha$ and $\beta$ such that $\alpha:=\sup _{t \geq t_{0}} \alpha(t)<$ 0 and $\beta:=\sup _{t \geq t_{0}} \beta(t)<-\alpha$. Then, system (2.8) satisfies the stability properties (2.4) and (2.5) by the results from [30] and [32], respectively. This conclusion, however, is just the special case from our results, as expressed in Remark 2.3.

3. The extended GL methods. The numerical methods we suggest for (2.1) will be based on GL methods for ODEs of the form $y^{\prime}(t)=f(t, y(t))$ with $y\left(t_{0}\right)=y_{0}$; see $[13,15,16]$. A GL method can be formulated as follows:

$$
\begin{cases}Y_{i}^{(n)}=h \sum_{j=1}^{s} c_{i j}^{(11)} f\left(t_{n}+c_{j} h, Y_{j}^{(n)}\right)+\sum_{j=1}^{r} c_{i j}^{(12)} y_{j}^{(n-1)}, & i=1,2, \ldots, s, \\ y_{i}^{(n)}=h \sum_{j=1}^{s} c_{i j}^{(21)} f\left(t_{n}+c_{j} h, Y_{j}^{(n)}\right)+\sum_{j=1}^{r} c_{i j}^{(22)} y_{j}^{(n-1)}, & i=1,2, \ldots, r\end{cases}
$$

with real coefficients $c_{i j}^{(I J)}$ for $I, J=1,2$, with a step size $h>0$, and with $t_{n}=t_{0}+n h$ for $n \geq 0$. The vector $Y_{i}^{(n)}$ approximates $y\left(t_{n}+c_{i} h\right)$. The precise nature of vector $y_{i}^{(n)}$ differs from method to method; generally, it contains the necessary information for doing the next time-integration step. For use in the subsequent analysis, we introduce the coefficient matrices $C_{I J}=\left(c_{i j}^{(I J)}\right)$ and the abscissa vector $c=\left(c_{1}, c_{2}, \ldots, c_{s}\right)^{T}$.

An important subclass of the GL methods, which will be considered further on, is the multistep Runge-Kutta (MRK) methods (see, e.g., [14, 24]):

$$
\left\{\begin{array}{l}
Y_{i}^{(n)}=h \sum_{j=1}^{s} a_{i j} f\left(t_{n}+c_{j} h, Y_{j}^{(n)}\right)+\sum_{j=1}^{r} \hat{a}_{i j} y_{n+j-1}, \quad i=1,2, \ldots, s, \\
y_{n+r}=h \sum_{j=1}^{s} b_{j} f\left(t_{n}+c_{j} h, Y_{j}^{(n)}\right)+\sum_{j=1}^{r} \hat{b}_{j} y_{n+j-1} .
\end{array}\right.
$$

These methods can be written in the form (3.1) when setting $y_{n+i}=y_{i}^{(n)}$. With $b=\left(b_{1}, b_{2}, \ldots, b_{s}\right)^{T}$ and $\hat{b}=\left(\hat{b}_{1}, \hat{b}_{2}, \ldots, \hat{b}_{r}\right)^{T}$, we can identify the coefficient matrices:

$$
C_{11}=\left(a_{i j}\right), \quad C_{12}=\left(\hat{a}_{i j}\right), \quad C_{21}=\left[\begin{array}{c}
0 \\
b^{T}
\end{array}\right], \quad C_{22}=\left[\begin{array}{cc}
0 & I_{r-1} \\
\hat{b}^{T}
\end{array}\right] .
$$

For the formulation of other numerical methods as a GL method, we refer the reader to $[16]$.

First, we recall a number of elementary concepts of GL methods that will be important for our stability analysis. 
Definition 3.1. Let $R(z)=C_{22}+z C_{21}\left(I_{s}-z C_{11}\right)^{-1} C_{12}$, where $z \in \mathbb{C}$ and where $I_{s}$ denotes the $s \times s$ identity matrix. A GL method is called strictly stable at infinity if $R(\infty):=\lim _{z \rightarrow \infty} R(z)$ exists and if its spectral radius satisfies $\rho(R(\infty))<1$.

Definition 3.2 (cf. [13]). A GL method is called $(k, l)$-algebraically stable if there exist real constants $k$ and $l$, a symmetric positive-definite matrix $G=\left(g_{i j}\right) \in \mathbb{R}^{r \times r}$, and a nonnegative diagonal matrix $D=\operatorname{diag}\left(d_{1}, d_{2}, \ldots, d_{s}\right) \in \mathbb{R}^{s \times s}$ such that matrix $M=\left(m_{i j}\right) \in \mathbb{R}^{(r+s) \times(r+s)}$ is nonnegative definite, with

$$
M=\left[\begin{array}{cc}
k G-C_{22}^{T} G C_{22}-2 l C_{12}^{T} G C_{12} & C_{12}^{T} D-C_{22}^{T} G C_{21}-2 l C_{12}^{T} D C_{11} \\
D C_{12}-C_{21}^{T} G C_{22}-2 l C_{11}^{T} D C_{12} & D C_{11}+C_{11}^{T} D-C_{21}^{T} G C_{21}-2 l C_{11}^{T} D C_{11}
\end{array}\right] .
$$

In particular, a (1,0)-algebraically stable method is called algebraically stable.

Adapting method (3.1) to MVIDE (2.1) and considering the case where the step size $h=\tau / m$ for $m$ a given positive integer yield the following numerical scheme:

$$
\begin{cases}Y_{i}^{(n)}=h \sum_{j=1}^{s} c_{i j}^{(11)} f\left(t_{n}+c_{j} h, Y_{j}^{(n)}, Y_{j}^{(n-m)}, Z_{j}^{(n)}\right)+\sum_{j=1}^{r} c_{i j}^{(12)} y_{j}^{(n-1)}, \quad i=1,2, \ldots, s, \\ y_{i}^{(n)}=h \sum_{j=1}^{s} c_{i j}^{(21)} f\left(t_{n}+c_{j} h, Y_{j}^{(n)}, Y_{j}^{(n-m)}, Z_{j}^{(n)}\right)+\sum_{j=1}^{r} c_{i j}^{(22)} y_{j}^{(n-1)}, \quad i=1,2, \ldots, r .\end{cases}
$$

$Z_{j}^{(n)}$ is an approximation to

$$
Z\left(t_{n}+c_{j} h\right):=\int_{t_{n-m}+c_{j} h}^{t_{n}+c_{j} h} g\left(t_{n}+c_{j} h, v, y(v)\right) d v
$$

and is computed by a convergent, compound quadrature formula

$$
Z_{j}^{(n)}=h \sum_{q=0}^{m} \nu_{q} g\left(t_{n}+c_{j} h, t_{n-q}+c_{j} h, Y_{j}^{(n-q)}\right), \quad j=1,2, \ldots, s .
$$

Such a quadrature formula can be derived from a uniform repeated rule (cf. $[3,9,33])$. For our stability analysis we need the rule to satisfy the following condition:

$$
h \sqrt{(m+1) \sum_{q=0}^{m}\left|\nu_{q}\right|^{2}}<\nu \quad \text { with } m h=\tau \text { and a positive constant } \nu .
$$

This condition holds for many of the common quadrature rules. For example, the compound trapezoidal (CT) rule of second order,

$$
\int_{0}^{\tau} \Phi(s) d s \cong h\left[\frac{1}{2} \Phi(0)+\sum_{q=1}^{m-1} \Phi((m-q) h)+\frac{1}{2} \Phi(m h)\right], \quad \text { where } m h=\tau,
$$

satisfies

$h \sqrt{(m+1) \sum_{q=0}^{m}\left|\nu_{q}\right|^{2}}=h \sqrt{(m+1)\left[(1 / 2)^{2}+m-1+(1 / 2)^{2}\right]} \leq h(m+1) \leq 2 m h=2 \tau$.

The compound Gregory (CG) rule of order 3 (cf. [9]),

$$
\int_{0}^{\tau} \Phi(s) d s \cong \frac{h}{12}\left[5 \Phi(0)+13 \Phi(h)+12 \sum_{q=2}^{m-2} \Phi((m-q) h)+13 \Phi((m-1) h)+5 \Phi(m h)\right],
$$


TABLE 1

Value for the bound $\nu$ in (3.5) for different compound quadrature rules.

\begin{tabular}{|c|c|c|c|c|}
\hline \hline & CT rule & CG rule & CS rule & CN rule \\
\hline$\nu$ & $2 \tau$ & $2 \tau$ & $\sqrt{20} \tau / 3$ & $2 \sqrt{1194} \tau / 45$ \\
\hline \hline
\end{tabular}

with $m h=\tau$, satisfies

$$
\begin{aligned}
h \sqrt{(m+1) \sum_{q=0}^{m}\left|\nu_{q}\right|^{2}} & =h \sqrt{(m+1)\left[\frac{5^{2}+13^{2}+(m-3) 12^{2}+13^{2}+5^{2}}{12^{2}}\right]} \\
& =h \sqrt{(m+1)\left(m-\frac{11}{36}\right)} \leq h(m+1) \leq 2 m h=2 \tau .
\end{aligned}
$$

The values for $\nu$ for the fourth order compound Simpson (CS) rule and for the sixth order compound Newton-Cotes (CN) rule have been derived in [33]; see Table 1.

We end this section with some more notational conventions. When the extended GL method is applied to system (2.1) with the initial function $\varphi(t)$ replaced by $\psi(t)$, the resulting numerical approximations will be denoted by $\tilde{Y}_{j}^{(n)}, \tilde{Z}_{j}^{(n)}$, and $\tilde{y}_{i}^{(n)}$. Also, when a time point $t_{n}$ or $t_{n}+c_{j} h$ falls in the initial interval $\left[t_{0}-\tau, t_{0}\right]$, we set the approximation at that point equal to the corresponding (known) true solution.

4. Some elementary lemmas. In this section, we will derive some technical lemmas, which are important for the derivation of the main results in the subsequent sections. First, we introduce some more notation:

$$
\begin{gathered}
\Delta y_{j}^{(n)}=y_{j}^{(n)}-\tilde{y}_{j}^{(n)}, \quad \Delta Y_{j}^{(n)}=Y_{j}^{(n)}-\tilde{Y}_{j}^{(n)}, \quad \Delta Z_{j}^{(n)}=Z_{j}^{(n)}-\tilde{Z}_{j}^{(n)}, \\
\Delta f_{j}^{(n)}=f\left(t_{j}^{(n)}, Y_{j}^{(n)}, Y_{j}^{(n-m)}, Z_{j}^{(n)}\right)-f\left(t_{j}^{(n)}, \tilde{Y}_{j}^{(n)}, \tilde{Y}_{j}^{(n-m)}, \tilde{Z}_{j}^{(n)}\right), \\
\Delta y^{(n)}=\left(\begin{array}{c}
\Delta y_{1}^{(n)} \\
\Delta y_{2}^{(n)} \\
\vdots \\
\Delta y_{r}^{(n)}
\end{array}\right), \quad \Delta Y^{(n)}=\left(\begin{array}{c}
\Delta Y_{1}^{(n)} \\
\Delta Y_{2}^{(n)} \\
\vdots \\
\Delta Y_{s}^{(n)}
\end{array}\right), \quad \Delta f^{(n)}=\left(\begin{array}{c}
\Delta f_{1}^{(n)} \\
\Delta f_{2}^{(n)} \\
\vdots \\
\Delta f_{s}^{(n)}
\end{array}\right) .
\end{gathered}
$$

With (3.3) we can write the relation between the above quantities compactly as

$$
\left\{\begin{array}{c}
\Delta Y^{(n)}=h\left(C_{11} \otimes I_{N}\right) \Delta f^{(n)}+\left(C_{12} \otimes I_{N}\right) \Delta y^{(n-1)}, \\
\Delta y^{(n)}=h\left(C_{21} \otimes I_{N}\right) \Delta f^{(n)}+\left(C_{22} \otimes I_{N}\right) \Delta y^{(n-1)},
\end{array}\right.
$$

where the symbol $\otimes$ denotes the Kronecker product. We will use the following inner product and norms on $\mathbb{C}^{N s}$ :

$$
\langle U, V\rangle=\sum_{i=1}^{s}\left\langle u_{i}, v_{i}\right\rangle, \quad\|U\|=\sqrt{\langle U, U\rangle}, \quad\|U\|_{G}=\sqrt{\langle U, G U\rangle},
$$

where vectors $U=\left(u_{1}^{T}, u_{2}^{T}, \ldots, u_{s}^{T}\right)^{T}, V=\left(v_{1}^{T}, v_{2}^{T}, \ldots, v_{s}^{T}\right)^{T} \in \mathbb{C}^{N s}$, and $u_{i}, v_{i} \in \mathbb{C}^{N}$. 
Lemma 4.1. Suppose that the GL method $(3.1)$ is $(k, l)$-algebraically stable with $0<k \leq \theta \leq 1$. Suppose that the quadrature formula (3.4) satisfies (3.5) and that the conditions (2.2) and (2.3) hold. Set $d=\sum_{j=1}^{s} d_{j}$. Then, method (3.3) satisfies

$$
\begin{aligned}
\left\|\Delta y^{(n)}\right\|_{G}^{2} \leq \theta^{n}\left\|\Delta y^{(0)}\right\|_{G}^{2}+2 \beta \tau \theta^{n-m} d \max _{-m \leq i \leq-1} \max _{1 \leq j \leq s}\left\{\left\|\Delta Y_{j}^{(i)}\right\|^{2}\right\} \\
+2\left[(h \alpha-l) \theta^{m}+h \beta\right] \sum_{i=0}^{n-1} \theta^{n-m-i-1} \sum_{j=1}^{s} d_{j}\left\|\Delta Y_{j}^{(i)}\right\|^{2} \\
+\frac{2 h \sigma(\nu \gamma)^{2}}{m+1} \sum_{i=0}^{n-1} \theta^{n-i-1} \sum_{j=1}^{s} \sum_{q=0}^{m} d_{j}\left\|\Delta Y_{j}^{(i-q)}\right\|^{2}, \quad n \geq 1 .
\end{aligned}
$$

Proof. It follows from a fairly straightforward (but tedious) computation and $(k, l)$-algebraic stability that (compare also $[13,20])$

$$
\begin{aligned}
\left\|\Delta y^{(n)}\right\|_{G}^{2}-k\left\|\Delta y^{(n-1)}\right\|_{G}^{2}-2 \sum_{j=1}^{s} d_{j} \Re\left\langle\Delta Y_{j}^{(n)}, h \Delta f_{j}^{(n)}-l \Delta Y_{j}^{(n)}\right\rangle & =-\sum_{i=1}^{r+s} \sum_{i=1}^{r+s} m_{i j}\left\langle\omega_{i}, \omega_{j}\right\rangle \\
& \leq 0,
\end{aligned}
$$

where $M=\left(m_{i j}\right)$, as defined in Definition 3.2, and

$$
\omega_{i}= \begin{cases}y_{i}^{(n-1)}, & 1 \leq i \leq r \\ h \Delta f_{i}^{(n)}, & r+1 \leq i \leq r+s\end{cases}
$$

This, together with the condition $0<k \leq \theta$, implies

$$
\left\|\Delta y^{(n)}\right\|_{G}^{2} \leq \theta\left\|\Delta y^{(n-1)}\right\|_{G}^{2}+2 \sum_{j=1}^{s} d_{j} \Re\left\langle\Delta Y_{j}^{(n)}, h \Delta f_{j}^{(n)}-l \Delta Y_{j}^{(n)}\right\rangle .
$$

By condition (2.2) one has

$$
\sum_{j=1}^{s} d_{j} \Re\left\langle\Delta Y_{j}^{(n)}, h \Delta f_{j}^{(n)}\right\rangle \leq h \sum_{j=1}^{s} d_{j}\left[\alpha\left\|\Delta Y_{j}^{(n)}\right\|^{2}+\beta\left\|\Delta Y_{j}^{(n-m)}\right\|^{2}+\sigma\left\|\Delta Z_{j}^{(n)}\right\|^{2}\right] .
$$

When this bound is inserted into (4.3), one finds

$$
\begin{aligned}
\left\|\Delta y^{(n)}\right\|_{G}^{2} \leq & \theta\left\|\Delta y^{(n-1)}\right\|_{G}^{2}+2(h \alpha-l) \sum_{j=1}^{s} d_{j}\left\|\Delta Y_{j}^{(n)}\right\|^{2} \\
& +2 h \beta \sum_{j=1}^{s} d_{j}\left\|\Delta Y_{j}^{(n-m)}\right\|^{2}+2 h \sigma \sum_{j=1}^{s} d_{j}\left\|\Delta Z_{j}^{(n)}\right\|^{2} .
\end{aligned}
$$

An induction argument applied to (4.4) yields

$$
\begin{aligned}
\left\|\Delta y^{(n)}\right\|_{G}^{2} \leq & \theta^{n}\left\|\Delta y^{(0)}\right\|_{G}^{2}+2(h \alpha-l) \sum_{i=0}^{n-1} \theta^{n-i-1} \sum_{j=1}^{s} d_{j}\left\|\Delta Y_{j}^{(i)}\right\|^{2} \\
& +2 h \beta \sum_{i=0}^{n-1} \theta^{n-i-1} \sum_{j=1}^{s} d_{j}\left\|\Delta Y_{j}^{(i-m)}\right\|^{2}+2 h \sigma \sum_{i=0}^{n-1} \theta^{n-i-1} \sum_{j=1}^{s} d_{j}\left\|\Delta Z_{j}^{(i)}\right\|^{2} .
\end{aligned}
$$


With (2.3), (3.5), and the Cauchy inequality, we have

$$
\begin{aligned}
\left\|\Delta Z_{j}^{(i)}\right\|^{2} & \leq(h \gamma)^{2}\left(\sum_{q=0}^{m}\left|\nu_{q}\right|\left\|\Delta Y_{j}^{(i-q)}\right\|\right)^{2} \leq(h \gamma)^{2}\left(\sum_{q=0}^{m}\left|\nu_{q}\right|^{2}\right)\left(\sum_{q=0}^{m}\left\|\Delta Y_{j}^{(i-q)}\right\|^{2}\right) \\
& \leq \frac{(\gamma \nu)^{2}}{m+1} \sum_{q=0}^{m}\left\|\Delta Y_{j}^{(i-q)}\right\|^{2} .
\end{aligned}
$$

Moreover, we have

$$
\begin{aligned}
& h \sum_{i=0}^{n-1} \theta^{n-i-1} \sum_{j=1}^{s} d_{j}\left\|\Delta Y_{j}^{(i-m)}\right\|^{2} \\
& \quad=h \sum_{i=0}^{n-m-1} \theta^{n-m-i-1} \sum_{j=1}^{s} d_{j}\left\|\Delta Y_{j}^{(i)}\right\|^{2}+h \sum_{i=-m}^{-1} \theta^{n-m-i-1} \sum_{j=1}^{s} d_{j}\left\|\Delta Y_{j}^{(i)}\right\|^{2} \\
& \quad \leq h \sum_{i=0}^{n-1} \theta^{n-m-i-1} \sum_{j=1}^{s} d_{j}\left\|\Delta Y_{j}^{(i)}\right\|^{2}+\tau \theta^{n-m} d \max _{-m \leq i \leq-1} \max _{1 \leq j \leq s}\left\{\left\|\Delta Y_{j}^{(i)}\right\|^{2}\right\} .
\end{aligned}
$$

Substituting (4.6) and (4.7) into (4.5) results in (4.2).

The second lemma gives an existence condition for the stability function $R(z)$ that is referred to in Definition 3.1.

Lemma 4.2. Suppose that a GL method is $(k, l)$-algebraically stable for a symmetric positive-definite matrix $G \in \mathbb{R}^{r \times r}$ and a positive diagonal matrix $D \in \mathbb{R}^{s \times s}$. Then the limit $R(\infty):=\lim _{z \rightarrow \infty} R(z)$ exists.

Proof. It suffices to prove the existence of $C_{0}:=\lim _{\varepsilon \rightarrow 0} C_{21}\left(C_{11}+\varepsilon I_{s}\right)^{-1}$. This can be shown in a similar way as Lemma 3.8 of [20] is proven (compare also [18]), i.e., by considering the nonnegative definiteness of the bottom right-hand principal minor $D C_{11}+C_{11}^{T} D-C_{21}^{T} G C_{21}-2 l C_{11}^{T} D C_{11}$ of matrix $M$.

REMARK 4.3. In $[18,20]$ the positivity of the diagonal matrix $D$ is guaranteed by requiring the underlying methods to be irreducible and algebraically stable. Here, we loosen these conditions by requesting positivity for $D$ explicitly. This allows us to consider more general $(k, l)$-algebraically stable $G L$ methods.

REMARK 4.4. When the conditions in Lemma 4.2 are satisfied, it holds that

$$
R(\infty)=\left\{\begin{aligned}
C_{22}-C_{21} C_{11}^{-1} C_{12} & \text { if } C_{11} \text { is invertible } \\
C_{22}-C_{0} C_{12} & \text { if } C_{11} \text { is singular. }
\end{aligned}\right.
$$

Two inequalities from [34] will also play important roles in the subsequent sections.

Lemma 4.5. Suppose that $\left\{A_{i}\right\}_{i=0}^{n}$ and $\left\{B_{i}\right\}_{i=-m}^{n}$ are two arbitrary nonnegative real sequences. Then the following two inequalities hold for all $n, m \geq 0$ :

$$
\begin{aligned}
\sum_{i=0}^{n}\left(A_{i} \sum_{j=0}^{m} B_{i-j}\right) & \leq \sum_{j=0}^{m} \sum_{i=0}^{n} A_{i+j} B_{i}+\left(\sum_{j=1}^{m} \sum_{i=1}^{j} A_{j-i}\right) \max _{-m \leq q \leq-1}\left\{B_{q}\right\}, \\
\sum_{i=0}^{n} \sum_{j=0}^{m} B_{i-j} & \leq(m+1) \sum_{i=0}^{n} B_{i}+\frac{m(m+1)}{2} \max _{-m \leq q \leq-1}\left\{B_{q}\right\} .
\end{aligned}
$$


Finally, we mention an asymptotic property of vector difference equations that can be derived easily from a combination of Theorems 105B and 123D of [16].

Lemma 4.6. Given a matrix $\mathcal{A} \in \mathbb{C}^{Q \times Q}$ and a sequence $V_{n} \in \mathbb{C}^{Q}$, the solution sequence $\left\{X_{n}\right\} \subseteq \mathbb{C}^{Q}$ of the linear difference equation $X_{n}=\mathcal{A} X_{n-1}+V_{n}$ satisfies $\lim _{n \rightarrow \infty}\left\|X_{n}\right\|=0$ if and only if $\rho(\mathcal{A})<1$ and $\lim _{n \rightarrow \infty}\left\|V_{n}\right\|=0$.

5. Global stability of the extended GL methods. Numerical stability is an important feature of an effective numerical method. An unstable numerical method may be consistent of high order, yet arbitrarily small perturbations will eventually cause large deviations from the true solution. In this section, we will focus on the global stability of the extended GL methods.

DeFinition 5.1. The extended GL method (3.3) and (3.4) is called globally stable for problems of class $\mathbb{G} \mathbb{R} \mathbb{I}(\alpha, \beta, \sigma, \gamma)$ if there exists a constant $\mathcal{H}>0$, which depends only on $\alpha, \beta, \sigma, \gamma, \tau, \nu$, and the method, such that

$$
\left\|\Delta y^{(n)}\right\| \leq \mathcal{H} \max \left\{\left\|\Delta y^{(0)}\right\|, \max _{-m \leq i \leq-1} \max _{1 \leq j \leq s}\left\{\left\|\Delta Y_{j}^{(i)}\right\|\right\}\right\} \quad \forall n \geq 1 .
$$

REMARK 5.2. For the extended one-step RK methods, we have $\Delta y^{(n)}=\Delta y_{n}:=$ $y_{n+1}-\tilde{y}_{n+1}$. Also, under the usual assumption that $0 \leq c_{i} \leq 1$, it holds that

$$
\max \left\{\left\|\Delta y_{0}\right\|, \max _{-m \leq i \leq-1} \max _{1 \leq j \leq s}\left\{\left\|\Delta Y_{j}^{(i)}\right\|\right\}\right\} \leq \max _{t_{0}-\tau \leq t \leq t_{0}}\|\varphi(t)-\psi(t)\| .
$$

Hence, we have for (5.1) the following equivalent formulation:

$$
\left\|\Delta y_{n}\right\| \leq \mathcal{H} \max _{t_{0}-\tau \leq t \leq t_{0}}\|\varphi(t)-\psi(t)\|
$$

Thus, the concept of global stability in Definition 5.1 is slightly stronger than that in [34].

THEOREM 5.3. Suppose that the GL method (3.1) is ( $k, l)$-algebraically stable for a nonnegative diagonal matrix $D=\operatorname{diag}\left(d_{1}, d_{2}, \ldots, d_{s}\right) \in \mathbb{R}^{s \times s}$ and a symmetric positive-definite matrix $G=\left(g_{i j}\right) \in \mathbb{R}^{r \times r}$, where $0<k \leq 1$, and suppose the quadrature formula (3.4) satisfies condition (3.5). Then the extended GL method (3.3) and (3.4) is globally stable for the class $\mathbb{G} \mathbb{R I}(\alpha, \beta, \sigma, \gamma)$ with stability constant

$$
\mathcal{H}=\sqrt{\frac{\lambda_{\text {max }}^{G}+d \tau\left(2 \beta+\sigma \gamma^{2} \nu^{2}\right)}{\lambda_{\text {min }}^{G}}},
$$

where $\lambda_{\min }^{G}$ and $\lambda_{\max }^{G}$ denote the minimum and maximum eigenvalues of matrix $G$ and $d=\sum_{j=1}^{s} d_{j}$, when the following condition holds:

$$
h\left(\alpha+\beta+\sigma \gamma^{2} \nu^{2}\right) \leq l .
$$

Proof. Setting $\theta=1$ in (4.2) gives

$$
\begin{aligned}
& \left\|\Delta y^{(n)}\right\|_{G}^{2} \leq\left\|\Delta y^{(0)}\right\|_{G}^{2}+2 \beta \tau d \max _{-m \leq i \leq-1} \max _{1 \leq j \leq s}\left\{\left\|\Delta Y_{j}^{(i)}\right\|^{2}\right\} \\
& +2[h(\alpha+\beta)-l] \sum_{i=0}^{n-1} \sum_{j=1}^{s} d_{j}\left\|\Delta Y_{j}^{(i)}\right\|^{2}+\frac{2 h \sigma(\nu \gamma)^{2}}{m+1} \sum_{i=0}^{n-1} \sum_{j=1}^{s} \sum_{q=0}^{m} d_{j}\left\|\Delta Y_{j}^{(i-q)}\right\|^{2} .
\end{aligned}
$$


Also, by virtue of inequality (4.9), we have for $n \geq 1$

$$
\sum_{i=0}^{n-1} \sum_{q=0}^{m}\left\|\Delta Y_{j}^{(i-q)}\right\|^{2} \leq(m+1) \sum_{i=0}^{n-1}\left\|\Delta Y_{j}^{(i)}\right\|^{2}+\frac{m(m+1)}{2} \max _{-m \leq i \leq-1}\left\{\left\|\Delta Y_{j}^{(i)}\right\|^{2}\right\} .
$$

Inserting (5.5) into (5.4) and using the condition that $m h=\tau$ yield

$$
\begin{aligned}
\left\|\Delta y^{(n)}\right\|_{G}^{2} \leq & \left\|\Delta y^{(0)}\right\|_{G}^{2}+d \tau\left(2 \beta+\sigma \gamma^{2} \nu^{2}\right) \max _{-m \leq i \leq-1} \max _{1 \leq j \leq s}\left\{\left\|\Delta Y_{j}^{(i)}\right\|^{2}\right\} \\
& +2\left[h\left(\alpha+\beta+\sigma \gamma^{2} \nu^{2}\right)-l\right] \sum_{i=0}^{n-1} \sum_{j=1}^{s} d_{j}\left\|\Delta Y_{j}^{(i)}\right\|^{2} .
\end{aligned}
$$

With condition (5.3) this leads to

$$
\left\|\Delta y^{(n)}\right\|_{G}^{2} \leq\left\|\Delta y^{(0)}\right\|_{G}^{2}+d \tau\left(2 \beta+\sigma \gamma^{2} \nu^{2}\right) \max _{-m \leq i \leq-1} \max _{1 \leq j \leq s}\left\{\left\|\Delta Y_{j}^{(i)}\right\|^{2}\right\} .
$$

Considering that $\lambda_{\text {min }}^{G}\left\|\Delta y^{(n)}\right\|^{2} \leq\left\|\Delta y^{(n)}\right\|_{G}^{2}$ and that $\left\|\Delta y^{(0)}\right\|_{G}^{2} \leq \lambda_{\text {max }}^{G}\left\|\Delta y^{(0)}\right\|^{2}$, we arrive at stability inequality (5.1) with stability constant (5.2).

Example 5.1. As an application of Theorem 5.3, we consider an extended GL method with $s=1$ and $r=2$, induced by the combination of the two-step BDF method and the CT rule:

$$
\left\{\begin{array}{l}
Y^{(n)}=\frac{2}{3} h F^{(n)}+\left[\left(\frac{4}{3},-\frac{1}{3}\right) \otimes I_{N}\right] y^{(n-1)} \\
y^{(n)}=h\left[\left(\begin{array}{l}
\frac{2}{3} \\
0
\end{array}\right) \otimes I_{N}\right] F^{(n)}+\left[\left(\begin{array}{cc}
\frac{4}{3} & -\frac{1}{3} \\
1 & 0
\end{array}\right) \otimes I_{N}\right] y^{(n-1)},
\end{array}\right.
$$

where $Y^{(n)}=y_{n+2}, y_{n} \cong y\left(t_{n}\right), y^{(n)}=\left(y_{n+2}^{T}, y_{n+1}^{T}\right)^{T}$, and

$$
\begin{aligned}
& F^{(n)}=f_{n+2}:=f\left(t_{n+2}, Y^{(n)}, Y^{(n-m)}, Z^{(n)}\right), \\
& Z^{(n)}=h \sum_{q=0}^{m}{ }^{\prime \prime} g\left(t_{n+2}, t_{n+2-q}, Y^{(n-q)}\right),
\end{aligned}
$$

where the special summation symbol stands for a sum with the first and last term halved. By Table 1 we know that (5.8) satisfies (3.5) with $\nu=2 \tau$. In [15], it was shown that the two-step BDF method is $(1 /(1-2 l), l)$-algebraically stable with $l \leq 0$ for $D=1$ and the $2 \times 2$ real symmetric positive-definite matrix

$$
G:=\left(\begin{array}{cc}
(5-2 l) / 2 & -1 \\
-1 & 1 / 2
\end{array}\right) .
$$

Note that $0<k \leq 1$ whenever $l \leq 0$ and that there always exists an $l$ such that (5.3) holds whenever $\beta+\sigma \gamma^{2} \nu^{2} \leq-\alpha$. Hence, we may conclude that the method is globally stable for the class $\mathbb{G} \mathbb{R} \mathbb{I}(\alpha, \beta, \sigma, \gamma)$ whenever $\beta+\sigma \gamma^{2} \nu^{2} \leq-\alpha$. For the parameters in the formula of the stability constant, the following values are used:

$$
d=1, \quad \nu=2 \tau, \quad \lambda_{\min }^{G}=\frac{3-l-\sqrt{(l-2)^{2}+4}}{2}, \quad \lambda_{\max }^{G}=\frac{3-l+\sqrt{(l-2)^{2}+4}}{2}
$$


TABLE 2

l-value minimizing (5.9) and corresponding stability constant $\mathcal{H}$ of the extended two-step BDF method for the MVIDEs in (7.1) and (7.2).

\begin{tabular}{|c|cc|cc|}
\hline \hline & \multicolumn{2}{|c|}{ MVIDE (7.1) } & \multicolumn{2}{c|}{ MVIDE $(7.2)$} \\
\hline$m$ & $l$ & $\mathcal{H}$ & $l$ & $\mathcal{H}$ \\
\hline 8 & -0.2224 & 6.4650 & -0.0554 & 7.1616 \\
\hline 16 & -0.1112 & 6.8497 & -0.0277 & 7.2997 \\
\hline 32 & -0.0556 & 7.0912 & -0.0138 & 7.3733 \\
\hline 64 & -0.0278 & 7.2279 & -0.0069 & 7.4116 \\
\hline \hline
\end{tabular}

The range of $l$ is given by $h\left(\alpha+\beta+\sigma \gamma^{2} \nu^{2}\right) \leq l \leq 0$. In particular, when the extended two-step BDF method with a fixed step size $h$ is applied to a concrete problem of class $\mathbb{G R I}(\alpha, \beta, \sigma, \gamma)$, one should look for the value of $l$ that minimizes the stability constant $\mathcal{H}$. This leads one to consider the following constrained minimization problem:

$$
\begin{cases}\operatorname{minimize} & \mathcal{H}:=\sqrt{\frac{3-l+\sqrt{(l-2)^{2}+4}+4 \tau\left(\beta+2 \sigma \gamma^{2} \tau^{2}\right)}{3-l-\sqrt{(l-2)^{2}+4}}} \\ \text { subject to } & h\left(\alpha+\beta+\sigma \gamma^{2} \nu^{2}\right) \leq l \leq 0\end{cases}
$$

In section 7 results of numerical experiments will be reported for two concrete MVIDEs. The first of those, specified in (7.1), belongs to class $\mathbb{G} \mathbb{R} \mathbb{I}\left(-4, \frac{1}{2}, \frac{1}{2}, 1\right)$; the second one, specified in (7.2), belongs to class $\mathbb{G} \mathbb{R I}\left(-4+\frac{\sqrt{2}}{2}, \frac{\sqrt{2}}{4}, \frac{\sqrt{2}}{4}, 2\right)$. We have numerically solved problem (5.9) for these two examples. The $l$-value at which the minimum is obtained and the corresponding $\mathcal{H}$-value are given in Table 2 as a function of the parameter $m$, with $h=\tau / m$.

The method in the above example is also algebraically stable. For such methods, a general result can be derived immediately from Theorem 5.3.

Corollary 5.4. Suppose that GL method (3.1) is algebraically stable for a nonnegative diagonal matrix $D=\operatorname{diag}\left(d_{1}, d_{2}, \ldots, d_{s}\right) \in \mathbb{R}^{s \times s}$ and a symmetric positivedefinite matrix $G=\left(g_{i j}\right) \in \mathbb{R}^{r \times r}$, and suppose the quadrature formula (3.4) satisfies condition (3.5). Then the extended GL method (3.3) and (3.4) is globally stable for the class $\mathbb{G} \mathbb{R} I(\alpha, \beta, \sigma, \gamma)$ with stability constant (5.2) when the following condition holds:

$$
\beta+\sigma \gamma^{2} \nu^{2} \leq-\alpha .
$$

In [14], Burrage has derived a class of $s$-stage MRK methods of order $2 s$ of the form (3.2) that satisfy

$$
\left\{\begin{array}{c}
B(2 s), C(s), E(s) ; c_{i} \neq c_{j} \text { whenever } i \neq j \\
\sum_{j=1}^{r} \hat{b}_{j}=1, i=1,2, \ldots, s ; \hat{b}_{1}>0, \hat{b}_{j} \geq 0, j=2,3, \ldots, r
\end{array}\right.
$$

where $B(2 s), C(s)$, and $E(s)$ denote the usual order conditions. It was shown that these methods are algebraically stable for the matrices

$$
G:=\operatorname{diag}\left(\hat{b}_{1}, \hat{b}_{1}+\hat{b}_{2}, \ldots, \sum_{j=1}^{r} \hat{b}_{j}\right), \quad D:=\operatorname{diag}\left(b_{1}, b_{2}, \ldots, b_{s}\right) .
$$


TABLE 3

Coefficients of the fourth order MRK method from Example 5.2.

\begin{tabular}{|cccc|}
\hline \hline$a_{11}$ & $a_{12}$ & $a_{21}$ & $a_{22}$ \\
\hline 0.47790690818421 & 0.87165188291653 & -0.08663699023763 & 0.50361252124048 \\
\hline$\hat{a}_{11}$ & $\hat{a}_{12}$ & $\hat{a}_{21}$ & $\hat{a}_{22}$ \\
\hline 0.75576439912123 & 0.24423560087877 & 0.97380878183171 & 0.02619121816829 \\
\hline$b_{1}$ & $b_{2}$ & $\hat{b}_{1}$ & $\hat{b}_{2}$ \\
\hline 0.95532987568936 & 0.79063681672548 & $2 \sqrt{15}-7$ & $8-2 \sqrt{15}$ \\
\hline & $c_{1}$ & $c_{2}$ & \\
\hline & 1.59379439197950 & 0.44316674917114 & \\
\hline \hline
\end{tabular}

Obviously, those are positive-definite. Hence, (5.10) is sufficient as a condition for global stability of the corresponding extended GL method.

Example 5.2. By combining the fourth order MRK method from [14] with the CS quadrature rule of order 4, we can obtain an extended GL method with $r=2$ and $s=2$. It is written schematically below; for the values of the coefficients we refer the reader to Table 3 .

$$
\left\{\begin{array}{c}
Y^{(n)}=h\left[\left(\begin{array}{ll}
a_{11} & a_{12} \\
a_{21} & a_{22}
\end{array}\right) \otimes I_{N}\right] F^{(n)}+\left[\left(\begin{array}{ll}
\hat{a}_{11} & \hat{a}_{12} \\
\hat{a}_{21} & \hat{a}_{22}
\end{array}\right) \otimes I_{N}\right] y^{(n-1)}, \\
y^{(n)}=h\left[\left(\begin{array}{cc}
0 & 0 \\
b_{1} & b_{2}
\end{array}\right) \otimes I_{N}\right] F^{(n)}+\left[\left(\begin{array}{cc}
0 & 1 \\
\hat{b}_{1} & \hat{b}_{2}
\end{array}\right) \otimes I_{N}\right] y^{(n-1)},
\end{array}\right.
$$

where $Y^{(n)}=\left(Y_{1}^{(n)^{T}}, Y_{2}^{(n)^{T}}\right)^{T}$ and $y^{(n)}=\left(y_{n+1}{ }^{T}, y_{n+2}{ }^{T}\right)^{T}$, with $Y_{i}^{(n)} \cong y\left(t_{n}+c_{i} h\right)$ and $y_{n} \cong y\left(t_{n}\right)$, and

$$
F^{(n)}=\left(f\left(t_{n}+c_{1} h, Y_{1}^{(n)}, Y_{1}^{(n-m)}, Z_{1}^{(n)}\right)^{T}, f\left(t_{n}+c_{2} h, Y_{2}^{(n)}, Y_{2}^{(n-m)}, Z_{2}^{(n)}\right)^{T}\right)^{T} .
$$

$Z_{j}^{(n)}$ is computed by the CS rule with an even integer $m \geq 4$ :

$$
\begin{aligned}
Z_{j}^{(n)}=\frac{h}{3}\left[g\left(t_{n, j}, t_{n, j}, Y_{j}^{(n)}\right)+4 \sum_{q=1}^{m / 2} g\left(t_{n j}, t_{n-2 q+1, j}, Y_{j}^{(n-2 q+1)}\right)\right. \\
\left.\quad+2 \sum_{q=1}^{(m-2) / 2} g\left(t_{n, j}, t_{n-2 q, j}, Y_{j}^{(n-2 q)}\right)+g\left(t_{n, j}, t_{n-m, j}, Y_{j}^{(n-m)}\right)\right] .
\end{aligned}
$$

Here the notation $t_{n, j}$ is used as shorthand for $t_{n}+c_{j} h$. This rule satisfies (3.5) for $\nu=\frac{\sqrt{20}}{3} \tau$. Under condition (5.10) this extended MRK method is globally stable. The stability constant (5.2) can be computed using $d=1.7460, \nu=\frac{\sqrt{20}}{3} \tau, \lambda_{\min }^{G}=$ $2 \sqrt{15}-7$, and $\lambda_{\max }^{G}=1$.

In [24], the MRK methods that satisfy (5.11) are called MRK methods of the first class. Five more classes of MRK methods are identified by $\mathrm{Li}$ in the above reference. All of those are proven to be algebraically stable for the matrices (5.12). Hence, Corollary 5.4 is immediately applicable to the corresponding extended GL methods. 
TABLE 4

Coefficients of the third order MRK method from Example 5.3.

\begin{tabular}{|cccc|}
\hline \hline$a_{11}$ & $a_{12}$ & $a_{21}$ & $a_{22}$ \\
\hline 0.41623635782678 & 0.43372361014760 & -0.22963576074350 & 0.62694215804990 \\
\hline$\hat{a}_{11}$ & $\hat{a}_{12}$ & $\hat{a}_{21}$ & $\hat{a}_{22}$ \\
\hline 0.04995996797438 & 0.95004003202562 & 0.48484848484848 & 0.51515151515152 \\
\hline$b_{1}$ & $b_{2}$ & $\hat{b}_{1}$ & $\hat{b}_{2}$ \\
\hline 0.59227996965099 & 0.55772003034901 & 0.15 & 0.85 \\
\hline & $c_{1}$ & $c_{2}$ \\
\hline & 1.80000000000000 & 0.91245791245791 \\
\hline
\end{tabular}

Example 5.3. We present an example of the second MRK class. Using formulas (3.11)-(3.16) of [24], we compute the coefficients for a two-stage third order MRK method. Its combination with the CG rule of order 3 (cf. [9]) yields another extended GL method of the form (5.13). The coefficients are given in Table 4 . The $Z_{j}^{(n)}$-values are computed as follows:

$$
\begin{aligned}
Z_{j}^{(n)}=\frac{h}{12}[ & 5 g\left(t_{n, j}, t_{n, j}, Y_{j}^{(n)}\right)+13 g\left(t_{n, j}, t_{n-1, j}, Y_{j}^{(n-1)}\right) \\
& +12 \sum_{q=2}^{m-2} g\left(t_{n, j}, t_{n-q, j}, Y_{j}^{(n-q)}\right)+13 g\left(t_{n, j}, t_{n-m+1, j}, Y_{j}^{(n-m+1)}\right) \\
& \left.+5 g\left(t_{n, j}, t_{n-m, j}, Y_{j}^{(n-m)}\right)\right] .
\end{aligned}
$$

Since the corresponding underlying method is algebraically stable, and since the CG rule satisfies (3.5) with $\nu=2 \tau$, we immediately have global stability of the extended GL method under condition (5.11). The stability constant (5.2) can be computed using $d=1.1500, \nu=2 \tau, \lambda_{\min }^{G}=0.15$, and $\lambda_{\max }^{G}=1$.

6. Asymptotic stability of the extended GL methods. In this section, we focus on the concept of asymptotic stability.

DeFinition 6.1. The extended GL method (3.3) and (3.4) is called asymptotically stable for problems of class $\mathbb{G} \mathbb{R} \mathbb{I}(\alpha, \beta, \sigma, \gamma)$ if $\lim _{n \rightarrow \infty}\left\|\Delta y^{(n)}\right\|=0$.

THEOREM 6.2. Suppose that GL method $(3.1)$ is $(k, l)$-algebraically stable for a nonnegative diagonal matrix $D=\operatorname{diag}\left(d_{1}, d_{2}, \ldots, d_{s}\right) \in \mathbb{R}^{s \times s}$ and a real symmetric positive-definite matrix $G=\left(g_{i j}\right) \in \mathbb{R}^{r \times r}$, where $0<k<1$, and suppose the quadrature formula (3.4) satisfies (3.5). Then the extended GL method (3.3) and (3.4) is asymptotically stable for the class $\mathbb{G} \mathbb{R I}(\alpha, \beta, \sigma, \gamma)$ when the following condition holds:

$$
h\left(\alpha+\beta+\sigma \gamma^{2} \nu^{2}\right)<l .
$$

Proof. Define the quantity $\theta=\max \left\{k,\left[\frac{h\left(\beta+\sigma \gamma^{2} \nu^{2}\right)}{l-h \alpha}\right]^{\frac{1}{m}}\right\}$. From $0<k<1$ and (6.1) it follows that $0<k \leq \theta<1$. Hence, bound (4.2) from Lemma 4.1 holds. We will simplify its right-hand side. By using (4.8), we can derive the following bound: 


$$
\begin{aligned}
& h \sum_{i=0}^{n-1} \theta^{-i} \sum_{q=0}^{m}\left\|\Delta Y_{j}^{(i-q)}\right\|^{2} \\
& \quad \leq h\left[\sum_{q=0}^{m} \sum_{i=0}^{n-1} \theta^{-(i+q)}\left\|\Delta Y_{j}^{(i)}\right\|^{2}+\left(\sum_{q=1}^{m} \sum_{i=1}^{q} \theta^{-(q-i)}\right) \max _{-m \leq \hat{q} \leq-1}\left\{\left\|\Delta Y_{j}^{(\hat{q})}\right\|^{2}\right\}\right] \\
& \quad \leq(m+1) h \sum_{i=0}^{n-1} \theta^{-(i+m)}\left\|\Delta Y_{j}^{(i)}\right\|^{2}+m \tau \theta^{1-m} \max _{-m \leq i \leq-1}\left\{\left\|\Delta Y_{j}^{(i)}\right\|^{2}\right\} .
\end{aligned}
$$

Substituting (6.2) into the last term of the right-hand side of (4.2) yields

$$
\begin{aligned}
\left\|\Delta y^{(n)}\right\|_{G}^{2} \leq & \theta^{n}\left\|\Delta y^{(0)}\right\|_{G}^{2}+2 \tau \theta^{n-m} d\left[\beta+\frac{m \sigma \gamma^{2} \nu^{2}}{m+1}\right] \max _{-m \leq i \leq-1} \max _{1 \leq j \leq s}\left\{\left\|\Delta Y_{j}^{(i)}\right\|^{2}\right\} \\
& +2\left[(h \alpha-l) \theta^{m}+h\left(\beta+\sigma \gamma^{2} \nu^{2}\right)\right] \sum_{i=0}^{n-1} \theta^{n-m-i-1} \sum_{j=1}^{s} d_{j}\left\|\Delta Y_{j}^{(i)}\right\|^{2} .
\end{aligned}
$$

By the definition of $\theta$ and with the inequalities $h \alpha \leq h\left(\alpha+\beta+\sigma \gamma^{2} \nu^{2}\right)<l$, one finds

$$
(h \alpha-l) \theta^{m}+h\left(\beta+\sigma \gamma^{2} \nu^{2}\right) \leq 0 .
$$

This inequality, together with the knowledge that $0<\theta<1$ and (6.3), leads to $\lim _{n \rightarrow \infty}\left\|\Delta y^{(n)}\right\|_{G}=0$ or $\lim _{n \rightarrow \infty}\left\|\Delta y^{(n)}\right\|=0$.

REMARK 6.3. The major difference between Theorems 6.2 and 5.3 lies in the strict inequalities present in both $k<1$ and (6.1).

Example 6.1. As an illustration we consider the method (5.7) and (5.8) again. Recall that (5.8) satisfies (3.5) with $\nu=2 \tau$ and that the underlying GL method (5.7) is $(1 /(1-2 l), l)$-algebraically stable for any $l \leq 0$. Thus, we have $0<k<1$ whenever $l<0$. Moreover, there always exists an $l(<0)$ such that $h\left(\alpha+\beta+\sigma \gamma^{2} \nu^{2}\right)<l$ whenever $\beta+\sigma \gamma^{2} \nu^{2}<-\alpha$. Hence, the latter is sufficient as a condition for asymptotic stability.

From Theorem 6.2 , it is not immediately possible to derive a corollary that applies specifically for algebraically stable methods because of the condition $k<1$. To remedy this situation, we present an alternative approach for proving asymptotic stability, where the parameter $k$ will be allowed to take the value 1 .

TheOREM 6.4. Suppose that the GL method (3.1) is strictly stable at infinity and $(k, l)$-algebraically stable for a positive diagonal matrix $D=\operatorname{diag}\left(d_{1}, d_{2}, \ldots, d_{s}\right) \in$ $\mathbb{R}^{s \times s}$ and a real symmetric positive-definite matrix $G=\left(g_{i j}\right) \in \mathbb{R}^{r \times r}$, where $0<k \leq$ 1. Suppose the quadrature formula (3.4) satisfies (3.5). Then the extended GL method (3.3) and (3.4) is asymptotically stable for the class $\mathbb{G} \mathbb{R I}(\alpha, \beta, \sigma, \gamma)$ when (6.1) holds.

Proof. Inequality (5.6) can be written as

$$
\begin{aligned}
\left\|\Delta y^{(n)}\right\|_{G}^{2}+ & 2\left[l-h\left(\alpha+\beta+\sigma \gamma^{2} \nu^{2}\right)\right] \sum_{i=0}^{n-1} \sum_{j=1}^{s} d_{j}\left\|\Delta Y_{j}^{(i)}\right\|^{2} \\
\leq & \left\|\Delta y^{(0)}\right\|_{G}^{2}+d \tau\left(2 \beta+\sigma \gamma^{2} \nu^{2}\right) \max _{-m \leq i \leq-1} \max _{1 \leq j \leq s}\left\{\left\|\Delta Y_{j}^{(i)}\right\|^{2}\right\} .
\end{aligned}
$$

Since $D$ is a positive diagonal matrix and since (6.1) holds, we may conclude from (6.4) that $\lim _{n \rightarrow \infty}\left\|\Delta Y_{j}^{(n)}\right\|^{2}=0$ for $j=1,2, \ldots, s$, and thus

$$
\lim _{n \rightarrow \infty}\left\|\Delta Y^{(n)}\right\|=\lim _{n \rightarrow \infty} \sqrt{\sum_{j=1}^{s}\left\|\Delta Y_{j}^{(n)}\right\|^{2}}=0 .
$$


The remainder of the proof is divided into two parts. First, we consider the case where matrix $C_{11}$ is invertible. Then, by (4.1), we have

$$
\Delta y^{(n)}=\left[R(\infty) \otimes I_{N}\right] \Delta y^{(n-1)}+\left[C_{21} C_{11}^{-1} \otimes I_{N}\right] \Delta Y^{(n)},
$$

where $R(\infty)=C_{22}-C_{21} C_{11}^{-1} C_{12}$. Because of the strict stability at infinity, one has

$$
\rho\left[R(\infty) \otimes I_{N}\right]=\rho[R(\infty)]<1 .
$$

Therefore, applying Lemma 4.6 to (6.6) yields $\lim _{n \rightarrow \infty}\left\|\Delta y^{(n)}\right\|=0$.

Next, we consider the case that matrix $C_{11}$ is singular. We replace it in (6.6) by the invertible matrix $\left(C_{11}+\varepsilon I_{s}\right)$ and let $\varepsilon \rightarrow 0$. This gives

$$
\Delta y^{(n)}=\left[R(\infty) \otimes I_{N}\right] \Delta y^{(n-1)}+\left(C_{0} \otimes I_{N}\right) \Delta Y^{(n)},
$$

where $R(\infty)=C_{22}-C_{0} C_{12}$ and $C_{0}=\lim _{\varepsilon \rightarrow 0} C_{21}\left(C_{11}+\varepsilon I_{s}\right)^{-1}$, whose existence is ensured by Lemma 4.2. Another application of Lemma 4.6 completes the proof.

When the GL method satisfies algebraic stability, Theorem 6.4 can be simplified.

Corollary 6.5. Suppose that the GL method (3.1) is strictly stable at infinity and algebraically stable for a positive diagonal matrix $D=\operatorname{diag}\left(d_{1}, d_{2}, \ldots, d_{s}\right) \in \mathbb{R}^{s \times s}$ and a real symmetric positive-definite matrix $G=\left(g_{i j}\right) \in \mathbb{R}^{r \times r}$, and suppose the quadrature formula (3.4) satisfies (3.5). Then the extended $G L$ method (3.3) and (3.4) is asymptotically stable for the class $\mathbb{G R I}(\alpha, \beta, \sigma, \gamma)$ when the following condition holds:

$$
\beta+\sigma \gamma^{2} \nu^{2}<-\alpha
$$

Example 6.2. Consider the extended GL method from Example 5.2. A computation shows that $\rho[R(\infty)] \cong 0.9816<1$, which implies strict stability. So, the method is asymptotically stable for problem class $\mathbb{G} \mathbb{R} \mathbb{I}(\alpha, \beta, \sigma, \gamma)$ under condition (6.9).

Example 6.3. Consider the extended GL method from Example 5.3. Now, we find that $\rho[R(\infty)] \cong 0.6158<1$. Hence, also for this method (6.9) is a sufficient condition to guarantee asymptotic stability.

7. Numerical experiments and convergence. In the previous sections we have proven that the extended GL methods possess an excellent stability behavior under a set of suitable conditions. Naturally, one also wishes to find out the actual computational performance and their accuracy in particular. Therefore, we will apply the methods from Examples 5.1, 5.2, and 5.3 to two MVIDE systems. In order to measure the quality of the numerical methods, we introduce the following:

$$
\text { err }:=\left\|y\left(t_{n}\right)-y_{n}\right\|_{\infty}, \quad \varepsilon:=\max _{0 \leq n \leq N_{f}}\left\|y\left(t_{n}\right)-y_{n}\right\|_{\infty}, \quad p:=\ln (\varepsilon) / \ln (h),
$$

where $N_{f}$ is a positive integer such that $N_{f} h=T$ with $T$ being the length of the solution interval. The symbols err, $\varepsilon$, and $p$ denote the error at time point $t_{n}$, the maximum error over the interval, and the convergence order of the method, respectively.

We will pay particular attention to the estimate of the convergence order. Our numerical results will seem to indicate that the convergence order of the extended GL method equals the minimum of the orders of the underlying GL method and the quadrature rule. These results are not entirely unexpected (see, e.g., the discussion of the combined order problem in [9, section 3.4]), and will be verified through a number 

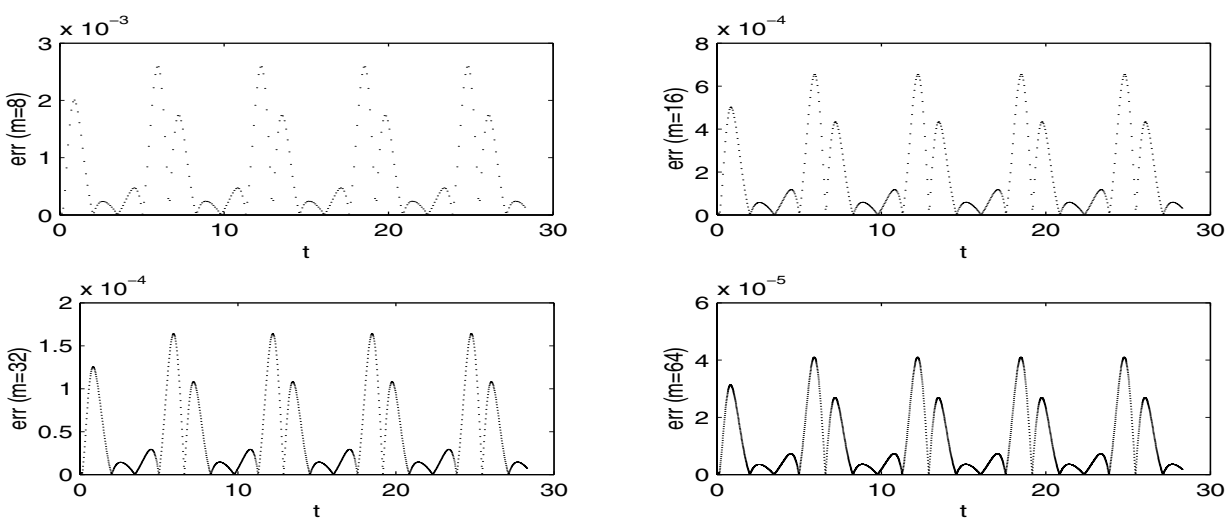

FiG. 7.1. Error of the method from Example 5.1 with $m=8,16,32,64$ for $(7.1)$ on $[0,9 \pi]$.

of examples. In the first two examples, the methods of Examples 5.1, 5.2, and 5.3 will be used. Those are characterized by a correct match between the orders of the GL method and the quadrature rule (order 2 for Example 5.1, order 3 for Example 5.3, and order 4 for Example 5.2). Further on, we will also consider methods where the order of the GL method is different from that of the quadrature rule.

Example 7.1. Consider the linear system with partially variable coefficients:

$$
y^{\prime}(t)=-(6+\sin t) y(t)+y\left(t-\frac{\pi}{4}\right)-\int_{t-\frac{\pi}{4}}^{t} \sin (v) y(v) d v+5 \exp (\cos t), t \geq 0
$$

with an initial condition on $[-\pi / 4,0]$ such that the exact solution is given by $y(t)=$ $\exp (\cos t)$. One may check that the problem belongs to class $\mathbb{G} \mathbb{R} I\left(-4, \frac{1}{2}, \frac{1}{2}, 1\right)$. With Theorem 2.1 we conclude that system (7.1) satisfies stability properties (2.4) and (2.5). Moreover, based on our earlier discussions, we deduce that the numerical methods from Examples 5.1, 5.2, and 5.3 retain the stability properties of the analytical solution; for this problem they are also globally and asymptotically stable.

In order to check the convergence behavior and accuracy of the extended GL methods, they will be applied using a sequence of step lengths characterized by the parameter $m$ on the time interval $[0,9 \pi]$. The accuracy of the obtained numerical solutions is displayed in Figures 7.1-7.3. The numerically estimated convergence orders are given in Table 5. The numerical results show that the methods are quite effective and preserve the inherent order of accuracy of the underlying components.

Example 7.2. Consider the following two-dimensional nonlinear system:

$$
\begin{aligned}
\frac{d}{d t}\left(\begin{array}{l}
y_{1}(t) \\
y_{2}(t)
\end{array}\right)= & -4\left(\begin{array}{l}
y_{1}(t) \\
y_{2}(t)
\end{array}\right)+\left(\begin{array}{cc}
0 & \sin t \\
\cos t & 0
\end{array}\right)\left(\begin{array}{l}
y_{1}\left(t-\frac{\pi}{5}\right) \\
y_{2}\left(t-\frac{\pi}{5}\right)
\end{array}\right) \\
& +\frac{1}{\sqrt{2}} \int_{t-\frac{\pi}{5}}^{t}\left(\begin{array}{c}
\frac{\left(1+\sin ^{2} v\right) y_{1}^{2}(v)}{1+y_{1}^{2}(v)} \\
\frac{\left(1+\cos ^{2} v\right) y_{2}^{2}(v)}{1+y_{2}^{2}(v)}
\end{array}\right) d v+\left(\begin{array}{c}
f(t) \\
g(t)
\end{array}\right), \quad 0 \leq t .
\end{aligned}
$$

Functions $f(t)$ and $g(t)$ and the initial condition on $[-\pi / 5,0]$ are chosen in such a way that the solution equals $y(t)=(\sin t, \cos t)^{T}$. This problem belongs to class $\mathbb{G} \mathbb{R I}\left(-4+\frac{\sqrt{2}}{2}, \frac{\sqrt{2}}{4}, \frac{\sqrt{2}}{4}, 2\right)$. It follows that $(7.2)$ satisfies stability properties $(2.4)$ and 

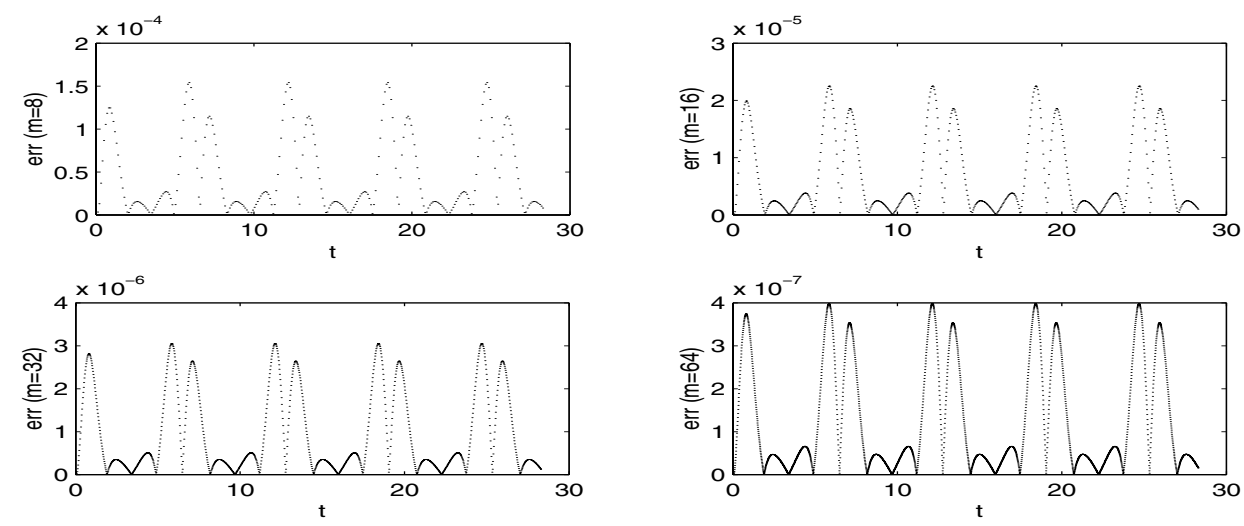

FiG. 7.2. Error of the method from Example 5.3 with $m=8,16,32,64$ for $(7.1)$ on $[0,9 \pi]$.
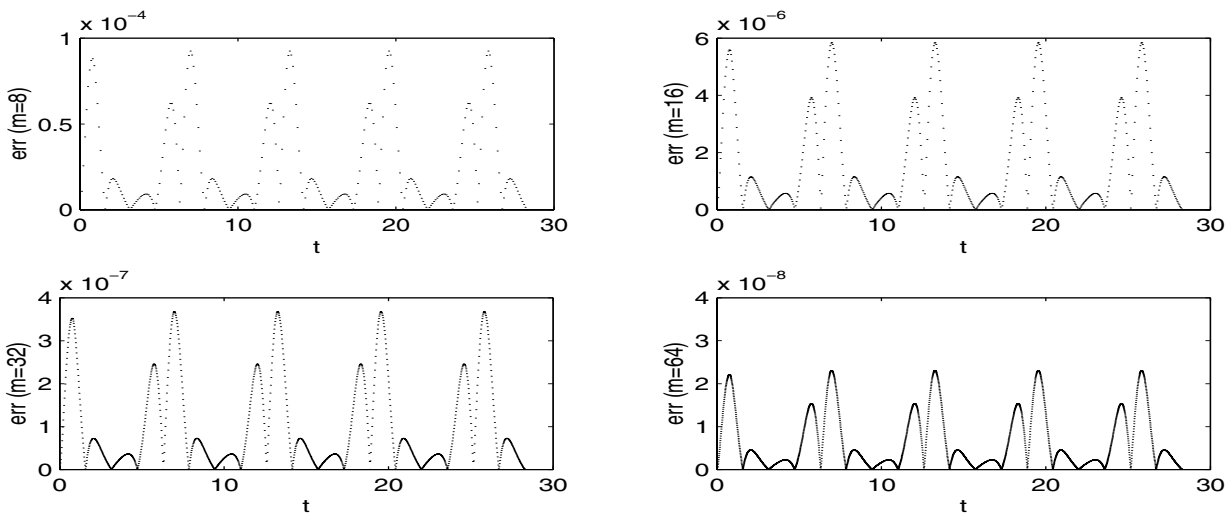

FIG. 7.3. Error of the method from Example 5.2 with $m=8,16,32,64$ for $(7.1)$ on $[0,9 \pi]$.

TABLE 5

Convergence order of extended GL methods for system (7.1). Example 5.1: 2nd order BDF + 2nd order quadrature; Example 5.3: 3rd order MRK + 3rd order quadrature; Example 5.2: 4th order $M R K+4$ th order quadrature.

\begin{tabular}{c|c|c|c}
\hline \hline$m$ & Example 5.1 & Example 5.3 & Example 5.2 \\
\hline 8 & 2.5666 & 3.7821 & 4.0022 \\
\hline 16 & 2.4327 & 3.5499 & 3.9982 \\
\hline 32 & 2.3510 & 3.4254 & 3.9964 \\
\hline 64 & 2.2956 & 3.3491 & 3.9960 \\
\hline \hline
\end{tabular}

(2.5) and that our three GL methods are globally and asymptotically stable. We consider the numerical results obtained when these methods are applied to (7.2). Note that a nonlinear equation is to be solved in every time-step. To that end, we adopted a Newton iteration, with initial values obtained by a backward interpolation formula

$$
\hat{Y}_{i}^{(n)}=y_{n+1}+\sum_{j=1}^{Q} \frac{1}{j !}\left(c_{i}-1\right) c_{i}\left(c_{i}+1\right)\left(c_{i}+2\right) \cdots\left(c_{i}+j-2\right) \nabla^{j} y_{n+1}, \quad i=1,2, \ldots,
$$



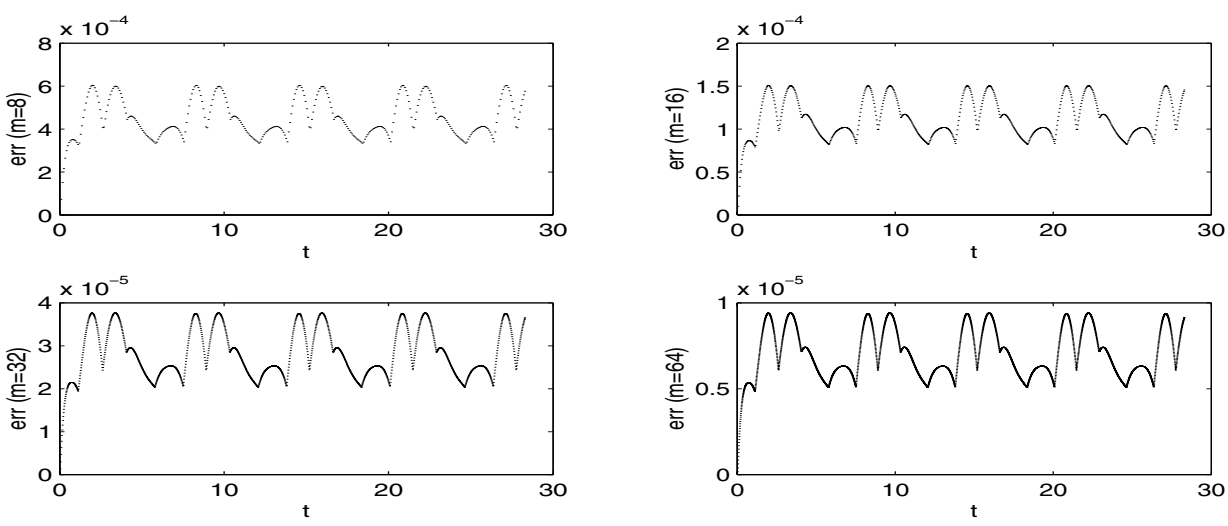

FIG. 7.4. Error of the method from Example 5.1 with $m=8,16,32,64$ for $(7.2)$ on $[0,9 \pi]$.
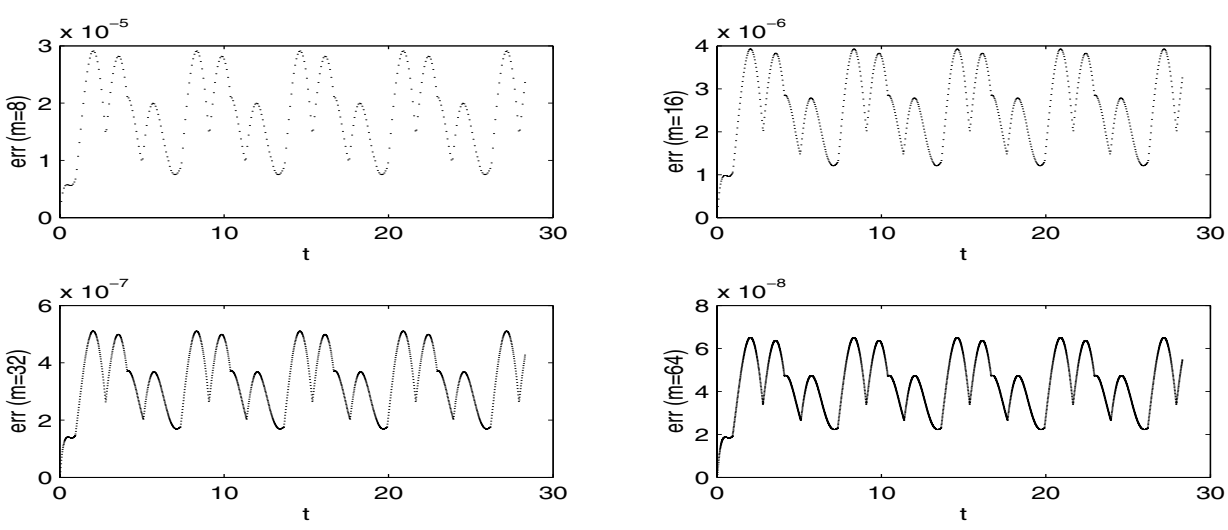

FiG. 7.5. Error of the method from Example 5.3 with $m=8,16,32,64$ for $(7.2)$ on $[0,9 \pi]$.

where $\hat{Y}_{i}^{(n)} \cong y\left(t_{n}+c_{i} h\right)\left(=y\left(t_{n+1}+\left(c_{i}-1\right) h\right)\right), 0<c_{i} \leq 2$. More precisely, in order to get a starting value with accuracy order matching the estimated order of the extended GL methods, we take $Q=2,3,4$ for the methods of Example 5.1, 5.3 , and 5.2, respectively. This proved to provide a great savings in computational cost. The accuracy of the numerical solutions are shown in Figures 7.4-7.6, and the estimated convergence orders are given in Table 6. As in the previous example, the methods again seem to preserve the inherent accuracy of the underlying components.

Example 7.3. Naturally, one also wishes to know what happens to an extended GL method when the order of the underlying method differs from that of the quadrature formula. In order to gain some insight, we considered the third order MRK method from Example 5.3 combined with the first order quadrature formula

$$
Z_{j}^{(n)}=h \sum_{q=0}^{m} g\left(t_{n, j}, t_{n-q, j}, Y_{j}^{(n-q)}\right), \quad j=1,2,
$$

combined with the second order quadrature formula

$$
Z_{j}^{(n)}=h \sum_{q=0}^{m} g\left(t_{n, j}, t_{n-q, j}, Y_{j}^{(n-q)}\right), \quad j=1,2,
$$



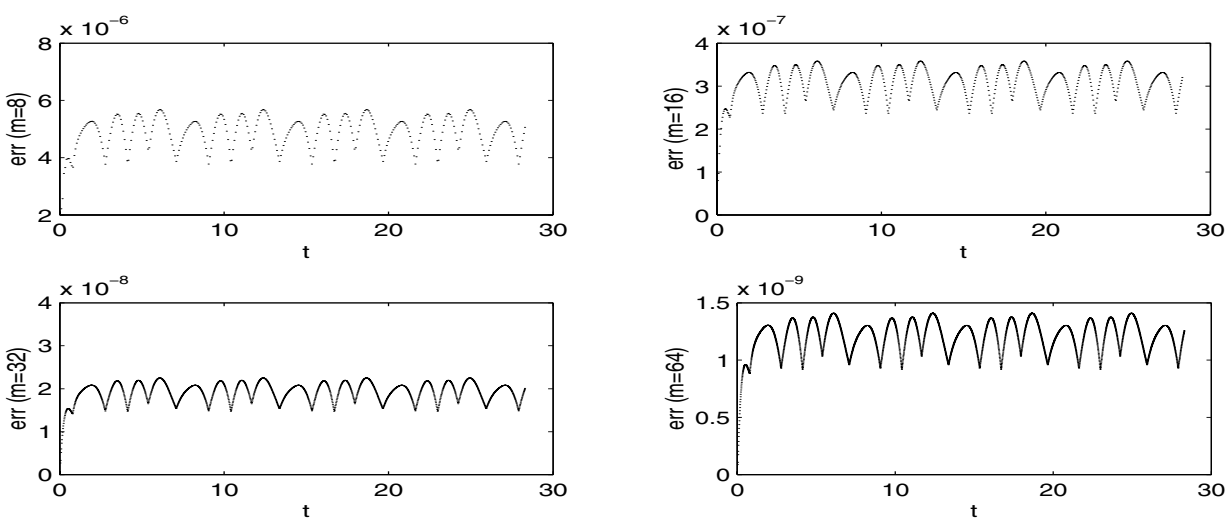

FiG. 7.6. Error of the method from Example 5.2 with $m=8,16,32,64$ for $(7.2)$ on $[0,9 \pi]$.

TABLE 6

Convergence order of extended GL methods for system (7.2). Example 5.1: 2nd order BDF + 2nd order quadrature; Example 5.3: 3rd order MRK + 3rd order quadrature; Example 5.2: 4th order $M R K+4$ th order quadrature.

\begin{tabular}{c|c|c|c}
\hline \hline$m$ & Example 5.1 & Example 5.3 & Example 5.2 \\
\hline 8 & 2.9132 & 4.1053 & 4.7479 \\
\hline 16 & 2.7183 & 3.8449 & 4.5847 \\
\hline 32 & 2.5918 & 3.6858 & 4.4804 \\
\hline 64 & 2.5029 & 3.5787 & 4.4078 \\
\hline \hline
\end{tabular}

TABLE 7

Convergence order of extended GL methods for (7.2): 3rd order MRK method combined with 1 st order method (7.3), combined with 2 nd order method (7.4), combined with 3rd order method (5.15), and combined with 4th order method (5.14).

\begin{tabular}{c|c|c|c|c}
\hline \hline$m$ & $(7.3)$ & $(7.4)$ & $(5.15)$ & $(5.14)$ \\
\hline 8 & 1.6318 & 3.5703 & 4.1053 & 4.2630 \\
\hline 16 & 1.4976 & 3.2562 & 3.8449 & 3.9556 \\
\hline 32 & 1.4102 & 3.0393 & 3.6858 & 3.7715 \\
\hline 64 & 1.3489 & 2.8821 & 3.5787 & 3.6492 \\
\hline \hline
\end{tabular}

and combined with the fourth order quadrature formula (5.14). The estimated convergence orders are given in Table 7 . For completeness, we also added the second column from Table 6 , where the method was combined with a third order quadrature method. Finally, in Table 8, we present similar results for the fourth order MRK method from Example 5.2 combined with four quadrature rules of different orders.

All of these results seem to indicate that the order of the extended GL method is equal to the minimum of the orders of its two components: the underlying GL method and the quadrature rule. A proof for this observation and a derivation of the conditions under which it would hold for class $\mathbb{G} \mathbb{R I}(\alpha, \beta, \sigma, \gamma)$ remain to be found.

8. Concluding remarks. We have investigated the stability of extended GL methods for a class of nonlinear MVIDEs. Numerical examples illustrate the convergence and the effectiveness of the methods. Our findings extend and improve 
TABLE 8

Convergence order of extended GL methods for (7.2): 4th order MRK method combined with 1 st order method (7.3), combined with 2 nd order method (7.4), combined with 3rd order method (5.15), and combined with 4th order method (5.14).

\begin{tabular}{c|c|c|c|c}
\hline \hline$m$ & $(7.3)$ & $(7.4)$ & $(5.15)$ & $(5.14)$ \\
\hline 8 & 1.6320 & 3.5895 & 4.3429 & 4.0022 \\
\hline 16 & 1.4976 & 3.2555 & 4.1125 & 3.9982 \\
\hline 32 & 1.4102 & 3.0354 & 3.9351 & 3.9964 \\
\hline 64 & 1.3489 & 2.8804 & 3.8030 & 3.9960 \\
\hline \hline
\end{tabular}

our earlier results reported in [33]. Specializing our results to the DDE $y^{\prime}(t)=f(t, y(t)$, $y(t-\tau)$ ), we find our results to be consistent with those in [20], slightly weaker than those in [30], and substantially different from those in $[28,29,6,7,31,32,25]$. As a conclusion, we would like to point out the precise differences.

In [30], the author studied the implicit Euler method for DDEs and proved that this method satisfies the following stability property:

$$
\left\|y_{n}-\tilde{y}_{n}\right\| \leq \max _{t_{0}-\tau \leq t \leq t_{0}}\|\varphi(t)-\psi(t)\| \quad \forall n \geq 0 .
$$

This strong property could be derived thanks to the relatively simple structure of the Euler scheme. The approach cannot be generalized to more complex methods.

In $[28,29]$, linear and nonlinear DDEs of class $\mathbb{G} \mathbb{R I}(-\rho, \rho, 0,0)$ with $\rho>0$ are considered. When an $A$-stable RK method is used in the linear case or an algebraically stable RK method is used in the nonlinear case, a specialized stability result can be derived, called $B_{\rho}$-stability. In particular, it is shown that

$$
\left\lceil y_{n+1}-\tilde{y}_{n+1}\right\rceil \leq\left\lceil y_{n}-\tilde{y}_{n}\right\rceil,
$$

where the notation $\lceil\cdot\rceil$ is defined as follows:

$$
\left\lceil y_{n}\right\rceil^{2}=\frac{1}{2}\left\|y_{n}\right\|^{2}+h \rho \sum_{n-m \leq j \leq n-1} \sum_{i=1}^{s} b_{i}\left\|y_{i}^{(j)}\right\| .
$$

This clearly shows the perturbation behavior of a combination of the numerical solution and the stage value approximations. In practice, however, one usually wishes to check the perturbation behavior of the numerical solution only.

In $[6,31]$, based on the concept of (semi-) $B N_{f}$-stability of the underlying continuous RK methods, the authors derived ( $R N-) G R N$-stability results for RK methods for DDEs, with a stability inequality characterized by (8.1). The special cases of continuous RK methods applied to linear autonomous and nonautonomous DDE problems are also considered. The concept of $B N_{f}$-stability is then specialized into that of $A_{f}$-stability and that of $A N_{f}$-stability, respectively. Under those conditions, (8.1) is obtained again. For more details and some extensions we refer the reader to [7]. The assumption of (semi-) $B N_{f}$-stability is rather strong, however, as it leads to an order barrier for the underlying RK methods. Indeed, in [32] it is shown that four is a sure order barrier for any $B N_{f}$-stable RK method. Moreover, at this moment, only two $B N_{f}$-stable RK methods have been found: the implicit Euler method and the Lobatto IIIC method with linear interpolation. Our research on the contrary is based on algebraic stability, which does not usually suffer from such a strong order barrier. 
In [25], Li studies RK methods applied to general Volterra functional differential equations. Under the condition of $B$-stability, it is shown that the numerical solutions $y_{n}$ and $\tilde{y}_{n}$ obtained from two different sets of initial data $\left\{\varphi(t), y_{1}, y_{2}, \ldots, y_{k}\right\}$ and $\left\{\psi(t), \tilde{y}_{1}, \tilde{y}_{2}, \ldots, \tilde{y}_{k}\right\}$ satisfy the following stability inequality:

$$
\left\|y_{n}-\tilde{y}_{n}\right\| \leq \exp \left(c\left(t_{n}-t_{k}\right)\right) \max \left\{\max _{1 \leq i \leq k}\left\|y_{i}-\tilde{y}_{i}\right\|, \max _{t_{0}-\tau \leq t \leq t_{0}}\|\varphi(t)-\psi(t)\|\right\},
$$

where $c$ is a positive constant and $h_{n}$ a variable step size. Note that the stability function $\mathcal{H}(t):=\exp \left(c\left(t-t_{k}\right)\right)$ in (8.3) is increasing for $t \geq t_{0}$ and will tend to infinity when $t \rightarrow+\infty$. Hence, (8.3) is applicable only on a finite time interval. Our stability results on the contrary can be applied on the infinite time interval since the stability function is a constant $\mathcal{H}$ and is independent of the time variable $t$.

\section{REFERENCES}

[1] C. T. H. BAKer AND N. J. Ford, Convergence of linear multistep methods for a class of delay integro-differential equations, in Numerical Mathematics, Singapore 1988, Internat. Schriftenreihe Numer. Math. 86, R. P. Agarwal, Y. M. Chow, and S. J. Wilson, eds., Birkhäuser, Basel, 1988, pp. 47-59.

[2] C. T. H. BAKer AND N. J. Ford, Asymptotic error expansions for linear multistep methods for a class of delay integro-differential equations, Bull. Soc. Math. Grèce (N.S.), 31 (1990), pp. $5-18$.

[3] C. T. H. BAKer AND N. J. Ford, Stability properties of a scheme for the approximate solution of a delay integro-differential equation, Appl. Numer. Math., 9 (1992), pp. 357-370.

[4] C. T. H. BAKer AND A. TANG, Generalized Halanay inequalities for Volterra functional differential equations and discrete versions, in Volterra Equations and Applications (Arlington, TX, 1996), Stability Control Theory Methods Appl. 10, C. Corduneanu and I. W. Sandberg, eds., Gordon and Breach, Amsterdam, 2000, pp. 39-55.

[5] C. T. H. BAKer AND N. J. Ford, Stability analysis of continuous Runge-Kutta methods for Volterra integro-differential systems with unbounded delays, Appl. Numer. Math., 24 (1997), pp. 153-173.

[6] A. Bellen And M. Zennaro, Strong contractivity properties of numerical methods for ordinary and delay differential equations, Appl. Numer. Math., 9 (1992), pp. 321-346.

[7] A. Bellen and M. Zennaro, Numerical Methods for Delay Differential Equations, Oxford University Press, Oxford, UK, 2003.

[8] F. Bloom, Ill-Posed Problems for Integrodifferential Equations in Mechanics and Electromagnetic Theory, SIAM Stud. Appl. Math. 3, SIAM, Philadelphia, 1981.

[9] H. Brunner and P. J. van Der Houwen, The Numerical Solution of Volterra Equations, CWI Monogr. 3, North-Holland, Amsterdam, 1986.

[10] H. Brunner, The numerical solution of neutral Volterra integro-differential equations with delay arguments, Ann. Numer. Math., 1 (1994), pp. 309-322.

[11] H. BRuNNER, The discretization of neutral functional integro-differential equations by collocation methods, Z. Anal. Anwendungen, 18 (1999), pp. 393-406.

[12] H. Brunner And R. Vermiglio, Stability of solutions of neutral functional integro-differential equations and their discretizations, Computing, 71 (2003), pp. 229-245.

[13] K. BurRage, Non-linear stability of a general class of differential equations methods, BIT, 20 (1980), pp. 185-203.

[14] K. Burrage, High order algebraically stable multistep Runge-Kutta methods, SIAM J. Numer. Anal., 24 (1987), pp. 106-115.

[15] J. C. Butcher, Stability properties for a general class of methods for ordinary differential equations, SIAM J. Numer. Anal., 18 (1981), pp. 37-44.

[16] J. C. Butcher, The Numerical Analysis of Ordinary Differential Equations: Runge-Kutta and General Linear Methods, John Wiley \& Sons, Chichester, UK, 1987.

[17] W. H. Enright And M. Hu, Continuous Runge-Kutta methods for neutral Volterra integrodifferential equations with delay, Appl. Numer. Math., 24 (1997), pp. 175-190.

[18] E. Hairer and M. Zennaro, On error growth of Runge-Kutta methods, Appl. Numer. Math., 22 (1996), pp. 205-216.

[19] C. Huang, H. Fu, S. Li, And G. Chen, Stability analysis of Runge-Kutta methods for nonlinear delay differential equations, BIT, 39 (1999), pp. 270-280. 
[20] C. Huang, S. Li, H. Fu, And G. Chen, Nonlinear stability of general linear methods for delay differential equations, BIT, 42 (2002), pp. 380-392.

[21] C. Huang and S. Vandewalle, Stability of Runge-Kutta-Pouzet Methods for Volterra Integral and Integro-differential Equations with Delays, Technical report TW 361, Department of Computer Science, Katholieke Universiteit Leuven, Leuven, Belgium, 2003.

[22] A. J. JERRI, Introduction to Integral Equations with Applications, Wiley-Interscience, New York, 1999.

[23] T. Кото, Stability of Runge-Kutta methods for delay integro-differential equations, J. Comput. Appl. Math., 145 (2002), pp. 483-492.

[24] S. LI, Stability and B-convergence properties of multistep Runge-Kutta methods, Math. Comp., 69 (1999), pp. 1481-1504.

[25] S. LI, B-theory of Runge-Kutta methods for stiff Volterra functional differential equations, Sci. China Ser. A, 46 (2003), pp. 662-674.

[26] P. Linz, Analytical and Numerical Methods for Volterra Equations, SIAM Stud. Appl. Math. 7, SIAM, Philadelphia, 1985.

[27] T. Luzyanina, K. Engelborghs, and D. Roose, Computing stability of differential equations with bounded and distributed delays, Numer. Algorithms, 34 (2003), pp. 41-66.

[28] J. Reverdy, Sur l'approximation d'équations d'évolution linéaires du premier ordre à retard par des méthodes de type Runge-Kutta, Thèse de doctorat, Université Paul Sabatier, Toulouse, France, 1981.

[29] J. Reverdy, Sur la B-stabilité pour une équation différentielle à retard, C. R. Acad. Sci. Paris Sér. I Math., 310 (1990), pp. 461-463.

[30] L. Torelli, Stability of numerical methods for delay differential equations, J. Comput. Appl. Math., 25 (1989), pp. 15-26.

[31] M. Zennaro, Contractivity of Runge-Kutta methods with respect to forcing terms, Appl. Numer. Math., 10 (1993), pp. 321-345.

[32] M. Zennaro, Asymptotic stability analysis of Runge-Kutta methods for nonlinear systems of delay differential equations, Numer. Math., 77 (1997), pp. 549-563.

[33] C. Zhang And S. Vandewalle, Stability analysis of delay-integro-differential equations and their backward differentiation time-discretization, J. Comput. Appl. Math., 164/165 (2004), pp. 797-814.

[34] C. Zhang And S. VAndewalle, Stability analysis of Runge-Kutta methods for nonlinear Volterra delay-integro-differential equations, IMA J. Numer. Anal., 24 (2004), pp. 193-214. 